\title{
1-S02-3 Symposium
}

\section{Physiological and pathophysiological relevance of zinc transporters}

\section{Toshiyuki Fukada $^{1}$}

${ }^{I}$ Tokushima Bunri Univ. Fac. of Pharm. Sci.

Zinc is an essential trace element that is required for a variety of cellular functions, and unbalanced zinc homeostasis results in health problems. Recent studies have highlighted that zinc acts as a signaling mediator: zinc signal, which is controlled via zinc transporters, and participates in health and disease conditions (1). In this symposium, I will address the updated information about the roles of zinc homeostasis and zinc signaling in physiology and pathophysiology.

The first manifestations that appear under zinc deficiency are skin defects (2). It should be also noted that about $60 \%$ of whole zinc in body is kept in skeletal muscle. In addition, attentions have been drawn to zinc accumulation in tumors. However, the physiological and pathophysiological relevance, and their zinc-related molecular mechanisms underlying normal skin and muscle development, as well as the mechanism by which disturbed zinc homeostasis causes disorders including cancers, have not been clarified yet.

In this symposium, I will provide an overview of the relationships between zinc dysregulation and skin disorders, by focusing on the roles of zinc transporter ZIP7 and ZIP10 in skin formation $(3,4)$, and cancer cachexia mediated by ZIP14 (5). I also address the zinc homeostatic system contributes skeletal muscle formation and function via zinc signaling mediated by ZIP13.

\section{References:}

1: International Journal of Medical Sciences 18: 2708, 2017

2: Nutrients 10: 219, 2018

3: Journal of Investigative Dermatology 137: 1682-1691, 2017

4: Proc. Natl. Acad. Sci. USA 114:12243-12248, 2017

5: Nature Medicine 24:770-781, 2018 